\title{
Special Issue on Alternative REALITY AND ANALYTICS FOR LEARNING: UNESCO-UNIR LATAM 2016
}

\section{Introduction}

One of the main challenges in modern education is to blend formal official learning with a huge variety of content provision, group discussion, self-entrepreneurship, user's own portfolio, and long lists of possibilities off-the-classroom. Informal learning happens anytime, anywhere. We strongly believe that formal learning does too. The cornerstone is to find the right balance between both contexts.

The effective integration of Open Educational Resources with formallessons, or between traditional lectures and live multi-user conferences, or even more, between spontaneous forum contributions outside the learning management system, and the official activities towards certification, will make the difference: the actual change of an educational paradigm. And this could be so for every single shareholder of the educational community, from teachers to tutors, from support staff to managers, from content providers to learning designers and, of course, from students to graduates.

This congress explores, analyses, discusses and, above all, suggests specific actions to take, in order to achieve a full integration of resources, learning styles, educational methodologies, administrative goals, institutional visions, and every single concern of each member of the Higher Education community. Researchers, teachers, experts and students from Russia, China, Germany, United Kingdom, Canada, Mexico, Puerto Rico, Ecuador, Colombia and Spain, have participated in the Congress UNESCOUNIR LATAM 2016, as a result, one may cite some of the many phrases expressed that will stand the test of time.

Alexander Khoroshilov, Director a.i. of UNESCO IITE (Institute for Information and Technology in Education, Moscow, Russia), stressed the idea that Educational Technology is essential to achieve the objectives in education that UNESCO 2030 has established: "The main goal is to ensure [an] education that offers opportunities for all, that is inclusive, and focuses on life-long learning. Something impossible if we do not have technology on our mindset".
Marisol Ramírez-Montoya, director of the unEsCo Chair "Open Educational Movement for Latin America", stated that "the specific actions proposed are intended to achieve innovation through the integration of resources, educational methodologies, administrative goals, institutional visions and internationalization on Higher Education". In this line, as pointed out by Daniel Burgos, president of the Congress, technology and methodology must go hand in hand: "Only an inclusive approach can allow us to integrate in the official programs what occurs outside the classrooms, in order to improve academic outcomes."

In this regard, the Congress proposed: systems for use, recycling and improved Open Education, methodologies that integrate neuroscience and creativity. It also instigates research on deep learning, mobile applications for literacy, possibilities of virtual reality and $3 \mathrm{D}$, and cyber-security.

\section{Project Quality}

The quality of the contributions received from different countries is highlighted. Everything has been reviewed by at least three members of the Academic Committee in a double-blind peer process. From the initial selection, the presentations made at the congress were evaluated and the best works were selected to invite them to send extended versions to our journal, which passed the double-blind review process again.

This special issue includes outstanding works and advances in Educational Technology, a discipline that evolves constantly, as can be seen in the selected papers that address proposed works. In this way, systems for the use, recycling and improvement of Open Educational Resources are also explored, in addition to methodologies that integrate neurosciences and creativity. Recommendations are collected for in-depth learning, mobile applications for literacy, the possibilities of virtual reality and 3D, and cyber-security. 


\section{Special Issue}

From papers selected by the UNIR ICT \& Education Program Committee for the Latam Congress 2016 (U2016), seven papers were chosen to be part of this special issue of the Journal. These articles are: "Method of Auto-configuration for Corporate Proxies", "Makerspaces in Libraries: Technology as Catalyst for Better Learning, Better Teaching", "Study of Student's Attitude Towards New Technological Contexts and Neuroscience Progress", "A Novel Framework to Use Association Rule Mining for Classification of Traffic Accident Severity", "Social Seducement: Towards the Foundations of a Pedagogical Model", "Engineering Students Academic Performance Prediction using ICFES Test Scores and Demographic Data", and "Possible Horizons for Educational Innovation in Colombia Through Contemporary Technological
Perspectives. A Training Experience with TIC. Contributions of the Project Analysis Model ReDCo CIER Occidente".

\section{Aknowledgements}

The editors would like to thank the Editorial Committee of the Ingeniería Solidaria Journal for hosting this special issue, the UNESCO IITE, the Universidad Internacional de La Rioja (UNIR), the UNESCO Chair in ELearning Department of the Vice-Rectorate for Research and Technology (UNIR Research) and the Universidad Cooperativa de Colombia, for the support of this special edition; and the U2016 Program Committee for its excellent work in the evaluation and selection of papers for the event.

Director, School of Engineering and Technology Universidad Internacional de La Rioja UNIR, Spain

E-mail: ruben.gonzalez@unir.net

Daniel Burgos

Vice-Rector of Research and Technology, Universidad Internacional de La Rioja UNIR, Spain.

E-mail: daniel.burgos@unir.net 


\section{EDICIÓN ESPECIAL SOBRE REALIDAD} ALTERNATIVA Y ANÁLISIS PARA EL APRENDIZAJE: UNESCO-UNIR LATAM 2016

\section{Introducción}

Uno de los principales retos de la educación moderna es combinar el aprendizaje formal oficial con una gran variedad de contenidos, discusiones en grupo, autoemprendimiento, el propio portafolio del usuario y una lista larga de posibilidades por fuera del aula. El aprendizaje informal ocurre en cualquier momento y en cualquier lugar; creemos firmemente que el aprendizaje formal también. La pieza clave es encontrar el equilibrio adecuado entre ambos contextos.

La integración efectiva de los Recursos Educativos Abiertos con lecciones formales o entre conferencias tradicionales y conferencias en vivo multiusuario o, incluso, entre contribuciones espontáneas de un foro por fuera del sistema de gestión del aprendizaje y las actividades oficiales en pos de la certificación, marcarán la diferencia: el cambio del paradigma educativo. Esto podría ser así para todos los involucrados en la comunidad educativa, desde maestros a tutores, desde el personal de apoyo hasta los directores, desde los proveedores de contenido hasta los diseñadores de aprendizaje y, por supuesto, desde los estudiantes hasta los graduados.

El Congreso UNESCO-UnIR LATAM 2016 explora, analiza, discute $y$, sobre todo, sugiere acciones específicas para lograr una integración plena de recursos, estilos de aprendizaje, metodologías educativas, objetivos administrativos, visiones institucionales y cada preocupación de cada miembro de la comunidad de la educación superior. Investigadores, profesores, expertos y estudiantes de Rusia, China, Alemania, Reino Unido, Canadá, México, Puerto Rico, Ecuador, Colombia y España participaron en este Congreso. Podríamos citar muchas de las frases expresadas al respecto que resistirán el paso del tiempo.

Alexander Khoroshilov, director provisional de UNESCO IITE (Instituto de Información y Tecnología en Educación, Moscú, Rusia), resaltó que la tecnología educativa es esencial para alcanzar los objetivos educativos establecidos por Unesco 2030: "El objetivo principal es asegurar [una] educación que ofrezca oportunidades para todos, que sea inclusiva y que se centre en el aprendizaje permanente, algo imposible de lograr si no introducimos la tecnología dentro de nuestra perspectiva”.

Marisol Ramírez-Montoya, directora de la Cátedra unesco "Movimiento Educativo Abierto para América Latina", señaló que "las acciones específicas propuestas pretenden lograr la innovación mediante la integración de recursos, metodologías educativas, objetivos administrativos, visiones institucionales y la internacionalización de la educación superior". En esta línea, como indicó Daniel Burgos, presidente del congreso, la tecnología y la metodología deben ir de la mano: "Solo un enfoque inclusivo nos permitirá integrar en los programas oficiales lo que ocurre por fuera de las aulas, para así poder mejorar los resultados académicos".

En este sentido, el Congreso propuso: sistemas de uso, reciclaje y mejora de la educación abierta, metodologías que integran la neurociencia y la creatividad. También instiga la investigación sobre el aprendizaje a fondo, las aplicaciones móviles para la alfabetización, las posibilidades de la realidad virtual y 3D, y la ciberseguridad.

\section{Calidad del proyecto}

Se destaca la calidad de las contribuciones recibidas de diferentes países. Todo ha sido revisado por al menos tres miembros del comité académico en un proceso de doble ciego. Desde la selección inicial, se evaluaron las presentaciones realizadas en el congreso y se eligieron las mejores obras para invitarlas a enviar versiones extendidas a nuestra revista, que luego pasaron nuevamente por el proceso de revisión doble ciego.

Esta edición especial incluye obras sobresalientes y avances en Tecnología Educativa, una disciplina que evoluciona constantemente, como se puede ver en los trabajos seleccionados que abordan las obras propuestas. De esta forma, se exploran también los sistemas de uso, reciclaje y mejora de los Recursos Educativos Abiertos, además de las metodologías que integran neurociencias y creatividad. Se recogen recomendaciones para el aprendizaje a fondo, las aplicaciones 
móviles para la alfabetización, las posibilidades de realidad virtual y $3 \mathrm{D}$, y ciberseguridad.

\section{Edición especial}

De los trabajos seleccionados por el UNIR ICT y el comité del programa educativo para el Congreso Latam 2016 (U2016), se eligieron siete trabajos para que formaran parte de esta edición especial de la revista. Estos artículos son: "Método de auto-configuración para proxies corporativos", "Makerspaces en las bibliotecas: La tecnología como catalizador para un mejor aprendizaje y una mejor enseñanza", "Estudio de la actitud estudiantil frente a nuevos contextos tecnológicos y el progreso de la neurociencia", "Nuevo marco para utilizar la minería de datos y reglas de asociación para la clasificación de la gravedad de accidentes de tráfico", "Seducción social: hacia los fundamentos de un modelo pedagógico", "La predicción del desempeño académico de los estudiantes de ingeniería usando los resultados de la prueba icFes y datos demográficos", y "Horizontes posibles para la innovación educativa en Colombia a través de perspectivas tecnológicas contemporáneas. Una experiencia de formación con Tic. Contribuciones del modelo de análisis de proyectos REDCO CIER Occidente".

\section{Agradecimientos}

Los editores desean agradecer al comité editorial de la revista Ingeniería Solidaria por haber acogido esta edición especial. Agradecerles a la UNESCO IITE, la Universidad Internacional de La Rioja (UNIR), la cátedra UNESCO en ELearning del vicerrectorado de Investigación y Tecnología (UNIR Research) y la Universidad Cooperativa de Colombia, por el apoyo brindado para esta edición especial; y al comité del programa del U2016 por su excelente trabajo en la evaluación y selección de trabajos para el evento.

Rubén González-Crespo

Director de la Escuela de Ingeniería y Tecnología, Universidad Internacional de La Rioja UNIR, España. Correo electrónico: ruben.gonzalez@unir.net

Daniel Burgos Vicerrector de Investigación y Tecnología, Universidad Internacional de La Rioja UNIR, España. Correo electrónico: daniel.burgos@unir.net 